\title{
Insula-Dorsal Anterior Cingulate Cortex Coupling is Associated with Enhanced Brain Reactivity to Smoking Cues
}

\author{
Amy C Janes*, ${ }^{*, 2}$, Stacey Farmer', Alyssa L. Peechatka', Blaise de B Frederick ${ }^{1,2}$ and Scott E Lukas ${ }^{1,2}$ \\ 'McLean Imaging Center, Brain Imaging Center, McLean Hospital, Belmont, MA, USA; ${ }^{2}$ Department of Psychiatry, Harvard Medical School, \\ Belmont, MA, USA
}

\begin{abstract}
The insula plays a critical role in maintaining nicotine dependence and reactivity to smoking cues. More broadly, the insula and the dorsal anterior cingulate cortex (dACC) are key nodes of the salience network (SN), which integrates internal and extrapersonal information to guide behavior. Thus, insula-dACC interactions may be integral in processing salient information such as smoking cues that facilitate continued nicotine use. We evaluated functional magnetic resonance imaging ( $\mathrm{fMRI}$ ) data from nicotine-dependent participants during rest, and again when they viewed smoking-related images. Greater insula-dACC coupling at rest was significantly correlated with enhanced smoking cue-reactivity in brain areas associated with attention and motor preparation, including the visual cortex, right ventral lateral prefrontal cortex, and the dorsal striatum. In an independent cohort, we found that insula-dACC connectivity was stable over I-h delay and was not influenced by changes in subjective craving or expired carbon monoxide, suggesting that connectivity strength between these regions may be a trait associated with heightened cue-reactivity. Finally, we also showed that insula reactivity to smoking cues correlates with a rise in cue-reactivity throughout the entire SN, indicating that the insula's role in smoking cue-reactivity is not functionally independent, and may actually represent the engagement of the entire SN. Collectively, these data provide a more networklevel understanding of the insula's role in nicotine dependence and shows a relationship between inherent brain organization and smoking cue-reactivity.

Neuropsychopharmacology (2015) 40, I56I-I568; doi:I0.1038/npp.20I5.9; published online I8 February 2015
\end{abstract}

\section{INTRODUCTION}

Exposure to smoking-related cues provides a major barrier to maintaining tobacco abstinence, as smoking cues motivate drug use and relapse (Ferguson and Shiffman, 2009). Functional magnetic resonance imaging (fMRI) has provided a wealth of information regarding the neural substrates of cue-reactivity, and a recent meta-analysis has identified several cortical brain regions as consistently playing a role in this process (Engelmann et al, 2012). One such cuereactive brain region is the insula, which is strongly linked to smoking behavior (Naqvi and Bechara, 2009) and addictive disorders more broadly (Naqvi et al, 2014). Specifically, cross-species research shows that nicotine use/seeking depends on an intact insula (Forget et al, 2010, Naqvi et al, 2007, Pushparaj et al, 2013), and greater insula reactivity to smoking cues predicts relapse vulnerability (Janes et al, 2010). More globally, the dorsal anterior insula (AI) is thought to have a critical role in 'developing and updating motivational states with specific associated actions' (Wager and Barret, 2004) and most likely provides information

\footnotetext{
*Correspondence: Dr AC Janes, McLean Imaging Center, Brain Imaging Center, McLean Hospital, Mail Stop 310, 115 Mill Street, Belmont, MA 02478, USA, Tel: +617855 3244, Fax: +617 8552770 ,

E-mail: ajanes@mclean.harvard.edu

Received 28 October 2014; revised 16 December 20।4; accepted 19 December 20।4; accepted article preview online 8 January 2015
}

necessary for goal-directed behavior (Nelson et al, 2010). For smokers, this suggests that the AI may be involved in linking the internal affective/motivational states triggered by smoking cues with action plans that maintain smoking.

However, it is unlikely that the AI's influence on behavior is functionally independent. In fact, during the majority of fMRI tasks resulting in insula activation, the dorsal anterior cingulate cortex (dACC) is co-activated, suggesting that these regions work together (Dosenbach et al, 2006). The relevance of AI-dACC interactions is highlighted by the fact that these regions are structurally (Allman et al, 2010) and functionally connected (Menon and Uddin, 2010). In fact, the AI and dACC are key nodes of the 'salience network' (SN), which is a well-defined functional brain network that integrates internal and extrapersonal information to guide behavior (Menon and Uddin, 2010). In regard to nicotine dependence, smoking cues, and the internal states they generate are highly salient 'internal and extrapersonal stimuli', which are known to elicit behavior such as relapse. However, it is still unclear whether AI-dACC interactions impact subsequent processing of salient smoking-related cues.

To evaluate the influence of AI-dACC interactions on brain reactivity to smoking cues, we took a number of approaches. First, we tested whether AI-dACC coupling strength at rest, during a task-free condition, influenced subsequent cue-reactivity. Resting-state functional connectivity is thought to represent inherent brain organization, which in turn influences brain function. For instance, 
functional connectivity at rest has been associated with mood states (Seeley et al, 2007), psychiatric, and neurological disorders (Menon, 2011), and age-related cognitive decline (Andrews-Hanna et al, 2007). We hypothesized that greater AI-dACC coupling may enhance salience processing and as a result, smoking cue-reactivity, possibly engaging brain activity patterns associated with behavioral responding to such salient stimuli. In an independent sample of tobacco smokers, we also evaluated the stability of AI-dACC connectivity over a 1-h delay and tested whether AI-dACC connectivity could be influenced by changes in subjective craving or expired carbon monoxide, which is a standard measure of recent smoking. This assessment will provide insight into whether AI-dACC connectivity strength is a stable trait, which persists over time or fluctuates as a function of short-term abstinence and changes in craving.

Finally, we evaluated whether bilateral AI reactivity to smoking cues correlated with a concurrent rise in smoking cue-reactivity throughout the entire SN. This analysis tested whether there is a functional link between the prominent role of the insula in smoking cue-reactivity (Janes et al, 2010) and the more global role of the SN in integrating internal and external stimuli to guide behavior. This goal was achieved by correlating the strength of insula reactivity to smoking $>$ neutral cues with smoking cue-reactivity in the whole brain. We then tested the hypothesis of whether this pattern of brain activity during cue-exposure overlapped with the SN as defined at rest. This second restingstate analysis was conducted using an independent component analysis (ICA), which is a data-driven approach used to define inherent brain organization and is unconstrained by any a priori predictions. Thus, we were able to directly compare the inherent organization of the SN at rest with activity patterns collected during smoking cue-presentation. Collectively, this work provides a more network-level understanding of the insula's role in smoking cue-reactivity and indicates how inherent brain organization impacts the processing of salient smoking cues. Such information will be useful to help identify the subset of patients who might respond to therapies aimed at reducing cue-reactivity as they undergo tobacco cessation treatment.

\section{MATERIALS AND METHODS}

\section{Participants}

Seventeen nicotine-dependent individuals (nine women/ eight men) participated in all study procedures at the McLean Imaging Center of McLean Hospital. Participants were an average of $31.4( \pm 6.1)$ years old, had $9.4( \pm 5.5)$ pack-years of cigarette use, reported smoking $>10$ cigarettes/day over the past 6 months, and were moderately to heavily nicotine dependent as indicated by a Fagerstrom test for nicotine dependence (FTND; Fagerström, 1978) score of $5.6( \pm 1.5)$. Subjective craving was measured just prior to scanning using the brief 10 -item form of the questionnaire for smoking urges (QSU; Cox et al, 2001). Participants were assessed by the structured clinical interview for DSM IV-TR and were excluded if they had a lifetime diagnosis of the following conditions: organic mental disorder, bipolar depression, and schizophrenia spectrum disorder. Participants were excluded for current substance use disorder other than nicotine dependence. Individuals were also excluded for current depressive episode, psychotropic drug use, pregnancy, and were required to have a zero blood alcohol level as measured by a breath sample (Alco-Sensor IV, Intoximeters, St Louis, MO). Recruitment was conducted using online advertisements and fliers posted in the Boston area. All participants gave verbal and written informed consent prior to participating in any study procedures, and the institutional review board at the McLean Hospital approved this study.

\section{Functional Neuroimaging}

All participants smoked one of their own cigarettes immediately after signing the informed consent to standardize the time since a cigarette was last smoked. MRI scanning began $\sim 1.5 \mathrm{~h}$ after smoking and expired carbon monoxide (CO; Micro Somkerlyzer II, Bedfont Scientific Instruments, Kent, UK) was measured immediately prior to scanning. Scans were acquired on a Siemens Trio $3 \mathrm{~T}$ scanner (Erlangen, Germany) with a 32-channel head coil. Multiecho multiplanar rapidly acquired gradient echo-structural images were acquired with the following parameters $(\mathrm{TR}=2.1 \mathrm{~s}$, TE $3.3 \mathrm{~ms}$, slices $=128$, matrix $=256 \times 256$, flip angle $=7^{\circ}$, resolution $=1.0 \times 1.0 \times 1.33 \mathrm{~mm}$ ). Gradient echo-planar images were collected during both resting state and cuereactivity. Slices were acquired aligned to the anterior and posterior commissures, and the phase encode direction was set to acquire from the posterior to anterior direction to prevent prefrontal signal loss. The 6-min resting-state acquisition used the following parameters $(T R=2.5 \mathrm{~s}$, $\mathrm{TE}=30 \mathrm{~ms}$, flip angle $=90^{\circ}$, slices $=42$, voxel size $=3.5 \mathrm{~mm}$ isotropic), while the cue-reactivity scan used the following parameters $\left(\mathrm{TR}=2 \mathrm{~s}, \mathrm{TE}=30 \mathrm{~ms}\right.$, flip angle $=75^{\circ}$, slices $=37$, distance factor $=10 \%$, voxel size $=3.5 \mathrm{~mm}$ isotropic, GRAP$\mathrm{PA}$ acceleration factor $=2$ ).

\section{Resting State}

The resting-state scan took place immediately prior to the cue-reactivity task to prevent any potential confound from cue-exposure. During the 6-min resting state, participants were asked to remain awake with their eyes open.

\section{Cue-Reactivity}

The cue-reactivity task was similar to our previous work (Janes et al, 2010). Participants were shown 60 smoking, 60 neutral, and 12 target images divided evenly across 5 blocks lasting $5 \mathrm{~min}$ and $18 \mathrm{~s}$ each (total task time $=26.5 \mathrm{~min}$ ). Images were presented for $4 \mathrm{~s}$ in a pseudorandom order (with no more than two of the same picture type occurring in a row) as performed in our prior study (Janes et al, 2010). Smoking images included smoking-related content such as people smoking, people holding cigarettes, or cigarettes alone. Neutral images were matched for content in that they involved people, hands, or objects such as pens or paintbrushes. These images were used in our prior work (Janes et al, 2013b). Target images were pictures of animals, and participants were asked to press a button each time a target image appeared. Inclusion of target images was to ensure that participants were awake and attended to the task. 
Images were separated by a jittered inter-trial-interval (ITI), where participants were shown a fixation cross on a black screen. The ITI times ranged from $6-14 \mathrm{~s}$ in intervals of $2 \mathrm{~s}$ with a $10 \mathrm{~s}$ average across block.

\section{fMRI Pre-Processing}

All data analysis was conducted using tools from the fMRI of the Brain (FMRIB) Software Library (FSL; www.fmrib.ox.ac.uk/fsl). The first five volumes for each run were removed to allow for signal stabilization. Functional data pre-processing included: motion correction with MCFLIRT, brain extraction using BET, slice timing correction, spatial smoothing with a Gaussian kernel of full-width half-maximum $6 \mathrm{~mm}$, and high-pass temporal filter with Gaussianweighted least-squares straight-line fitting with $\sigma=100 \mathrm{~s}$. An in-house written program was also used to detect and adjust for artifacts due to motion and intensity spiking. Subject specific data were registered to the MNI152 $2 \mathrm{~mm}^{3}$ standard space template (Montreal Neurological Institute, Montreal, QC, Canada), and the fMRI data were transformed into standard space at $2 \times 2 \times 2 \mathrm{~mm}$ resolution using the registration transformation matrices.

\section{Resting-State Analysis: denoising}

To prevent motion and other sources of noise from influencing resting-state analyses, each subject's data was denoised using FSL's multivariate exploratory linear decomposition into ICs (MELODIC). First, MELODIC was used to identify all ICs on an individual subject basis. The spatial maps and associated time courses for each of these ICs were then visually inspected and those ICs representing noise were identified (Kelly et al, 2010). The noise components, which could be due to participant motion or scanner artifact, were then regressed out of the fMRI data using FSL's fsl_regfilt utility.

\section{ICA: identification of SN}

To identify the SN, we used a group-level ICA, which is a data-driven approach for defining functional brain networks. First, data from all subjects were temporally concatenated and a multivariate group probabilistic ICA was conducted using FSL MELODIC. Consistent with our prior work (Janes et al, 2012, 2014a), the dimensionality was fixed to 35 to investigate large-scale resting-state networks. To ensure stable convergence of the ICA, the ICA was run eight times, followed by a meta-level ICA fed by all of the spatial maps from all eight individual runs. The SN was identified by visual inspection (Figure 2).

\section{AI-dACC Coupling at Rest}

To quantify AI-dACC coupling for each participant, average time courses for the bilateral AI region-of-interest (ROI) and midline ACC ROI were extracted from the denoised resting-state data. The bilateral AI ROI was comprised of two 5-mm spheres located at MNI coordinates $\pm 34,26,2$ ( $x$, $y$ and $z$, Supplementary Figure 1). This specific subregion of the AI was chosen based on a number of previously published studies showing co-activation of the ACC and AI
(Dosenbach et al, 2007, Ploran et al, 2007), and this ROI overlapped with our previous work (Janes et al, 2010, $2013 \mathrm{~b})$. The dACC ROI comprised of two $5-\mathrm{mm}$ spheres located at MNI coordinates $(0,32,20)$ and $(0,24,26)$, which fell into our previously defined AACC region (Janes et al, 2013a, Supplementary Figure 1). In addition, right AI-dACC coupling was significantly correlated with left AI-dACC coupling ( $r=0.65, p<0.004)$; accordingly bilateral insula coupling with the dACC was used for all analyses. As a follow-up analysis to test the specificity of AI-dACC connections on smoking cue-reactivity, we also evaluated the impact of bilateral mid $( \pm 8,2,8)$ and posterior insula $( \pm 38,-14,8)$ interactions with dACC (Supplementary Figure 1). The average time course for the insula and dACC ROIs were then demeaned, detrended, Hamming windowed (Oppenheim and Schafer, 1975, page 242), and correlated, resulting in one correlation value $(r)$ per participant.

\section{Cue-Reactivity Analysis}

The first-level analysis was conducted on each of the participant's five cue-reactivity runs separately. The first-level general linear model included three regressors corresponding to smoking, neutral, and target image presentation. These regressors were convolved with the gamma hemodynamic response function. Confound regressors also were included to model motion effects $(x, y, z$ translation and rotation motion). Contrasts were conducted between the smoking and neutral image conditions. First-level results were then combined (across the five blocks) using a secondlevel fixed effects analysis to generate the average brain reactivity for each individual participant. The group-level analysis was then conducted using a mixed model to identify the average brain reactivity across all participants. To evaluate the effect of AI-dACC coupling on cuereactivity, AI-dACC correlation values extracted from the resting-state data were demeaned across all participants and entered into the group-level analysis as a covariate. This same procedure was repeated for mid insula-dACC and posterior insula-dACC values. All results were corrected for multiple comparisons using a cluster threshold of $Z=2.3$, $p<0.05$.

To determine whether insula reactivity to smoking $>$ neutral cues is functionally associated with smoking > neutral activity throughout the $\mathrm{SN}$, we extracted beta-weights for the smoking > neutral contrast from the bilateral AI ROI defined above (Supplementary Figure 1). The beta-weights from the bilateral AI were demeaned and used as a covariate in a whole-brain analysis of smoking $>$ neutral cue-reactivity. The cluster thresholded results $(Z=2.3, p<0.05)$ from this analysis were then compared with the $\mathrm{SN}$ that was defined at rest. This was done using the FSL tool fslcc, which calculates the normalized spatial cross-correlation coefficient between these two spatial maps.

\section{Independent Cohort: stability of AI-dACC Connectivity}

To assess the stability of AI-dACC connectivity, we reanalyzed data from a separate cohort of 17 participants $(8 \mathrm{men} / 9$ women) who underwent two 6-min resting-state scans at the beginning and end of a 1-h scanning session. Data from this cohort was published previously (Janes et al, 2014a), 
and all relevant methods were identical to those described above. These participants were $25.4( \pm 4.6)$ years old with $15.3 \pm 2.1$ years of education, $6.7 \pm 4.7$ pack-years of smoking experience, and an average FTND score of $6.3 \pm 1.0$. To determine whether AI-dACC connectivity is impacted by a change in craving or expired $\mathrm{CO}$ over this 1 -h time period, a correlation analysis was conducted between a change in these measures post- $v s$ pre-scan and the change in connectivity (AI-dACC $r$-value from the second minus the first resting-state scan). Craving was measured as the sum of all questions on the QSU and both craving and $\mathrm{CO}$ were measured just before and after scanning.

\section{RESULTS}

\section{Resting-State Coupling and Demographics}

On average, all participants had positive coupling between the bilateral AI and AACC (average $r=0.64, \pm 0.15$ ). Restingstate AI-dACC interactions were not correlated with pre-scan CO levels, age, craving, FTND, or pack-year. Expired CO levels were $23.2 \pm 2.3$ p.p.m. just prior to scanning.

\section{Brain Reactivity to Smoking $>$ Neutral Cues}

On average, brain reactivity for the smoking $>$ neutral contrast was increased in cortical midline structures including the medial prefrontal cortex (mPFC), ACC, posterior cingulate cortex, and precuneus. Activation was also found in the bilateral angular gyrus and insula (Table 1; Figure 1; cluster-corrected $Z=2.3, p<0.05$ ).

\section{Insula Cue-Reactivity and the SN}

As insula reactivity to smoking cues increased so did the brain reactivity to smoking cues in regions traditionally defined as part of the SN. There was substantial overlap between this cue-reactivity activation pattern (red overlay) and the $\mathrm{SN}$ as defined by ICA at rest (blue overlay; $r=0.43$; Figure 2).

\section{Resting-State Coupling and Cue-Reactivity}

Dorsal AI-dACC interactions significantly influenced brain reactivity to smoking $>$ neutral cues. Specifically, stronger AI-dACC coupling at rest resulted in greater brain reactivity to smoking cues in the visual cortex, right ventrolateral PFC (VLPFC, Brodmann area (BA) 47), and the right putamen extending into the posterior insula (VLPFC, BA 47; Table 2; Figure 1; cluster-corrected $Z=2.3, p<0.05$ ). No association was found between cue-reactivity and bilateral mid or posterior insula interactions with the dACC.

\section{Stability of AI-ACC Connectivity}

In the separate cohort of participants, we found no change in AI-dACC connectivity over a 1 -h delay (paired $t$-test $t_{16}=, p=0.31, r-$ value at time $1: 0.61 \pm 0.19, r-$ value at time 2: $0.68 \pm 0.13$. Craving levels significantly increased over this delay $\left(t_{16}=-3.3, p<0.01\right)$, while expired CO levels decreased $\left(t_{16}=6.3, p<0.001\right)$. However, no association was found between the difference in AI-dACC connectivity and the change in craving or expired CO.

\section{DISCUSSION}

An intact insula is necessary for the maintenance of nicotine dependence in humans (Naqvi and Bechara, 2009) and drug seeking in rodents (Forget et al, 2010). While these prior studies focused exclusively on the insula, the current results provide a more network-level understanding of this brain region's involvement in smoking cue-reactivity. Not only does insula reactivity to smoking cues correspond with activity throughout the SN, but AI-dACC coupling at rest predicts enhanced subsequent brain reactivity to smoking cues. These findings indicate that the insula reactivity typically seen in response to smoking cues (Engelman et al, 2012) is not functionally independent, but may represent engagement of a larger network involved in salience processing.

Table I Brain Reactivity to Smoking $>$ Neutral Cues

\begin{tabular}{|c|c|c|c|c|c|c|}
\hline Brain area & $\begin{array}{l}\text { Brodmann } \\
\text { area }\end{array}$ & $x$ & y & $\mathbf{z}$ & Z-max & Voxels \\
\hline $\begin{array}{l}\text { Bilateral frontal pole extending into the superior frontal gyrus; aspects of the anterior cingulate } \\
\text { cortex; and anterior left middle frontal gyrus }\end{array}$ & $6,9,8,10,32$ & 8 & 22 & 64 & 4.48 & 9223 \\
\hline Bilateral posterior cingulate cortex extending into the precuneus & $7,23,31$ & -8 & -54 & 26 & 4.81 & 5311 \\
\hline Left angular and supramarginal gyri & $7,19,37,39$ & -48 & -60 & 30 & 4.82 & 4271 \\
\hline Right angular and supramarginal gyri & 19,39 & 60 & -60 & 24 & 4.4 & 1926 \\
\hline Right hypothalamus, bilateral pons, right superior colliculus, and right inferior colliculus & NA & 12 & -28 & -32 & 3.37 & 896 \\
\hline Anterior and posterior left insular cortex, extending into the posterior orbital gyrus and pars triangularis & 13,47 & -54 & 30 & -2 & 3.52 & 834 \\
\hline $\begin{array}{l}\text { Anterior aspects of the right inferior temporal gyrus, extending slightly into the right anterior middle } \\
\text { temporal gyrus and right anterior temporal pole }\end{array}$ & $20,38,21$ & 44 & 2 & -42 & 4.2 & 767 \\
\hline
\end{tabular}

Brain area and Brodmann area refer to the location of each cluster of contiguous voxels. MNI coordinates $(x, y, z)$ refer to the region of maximum cluster activation for each cluster. Z-max refers to the maximum Z-statistic in each cluster ( $p_{\text {cluster corrected }}<0.05$ ). Voxels refer to the total number of voxels within the cluster. 

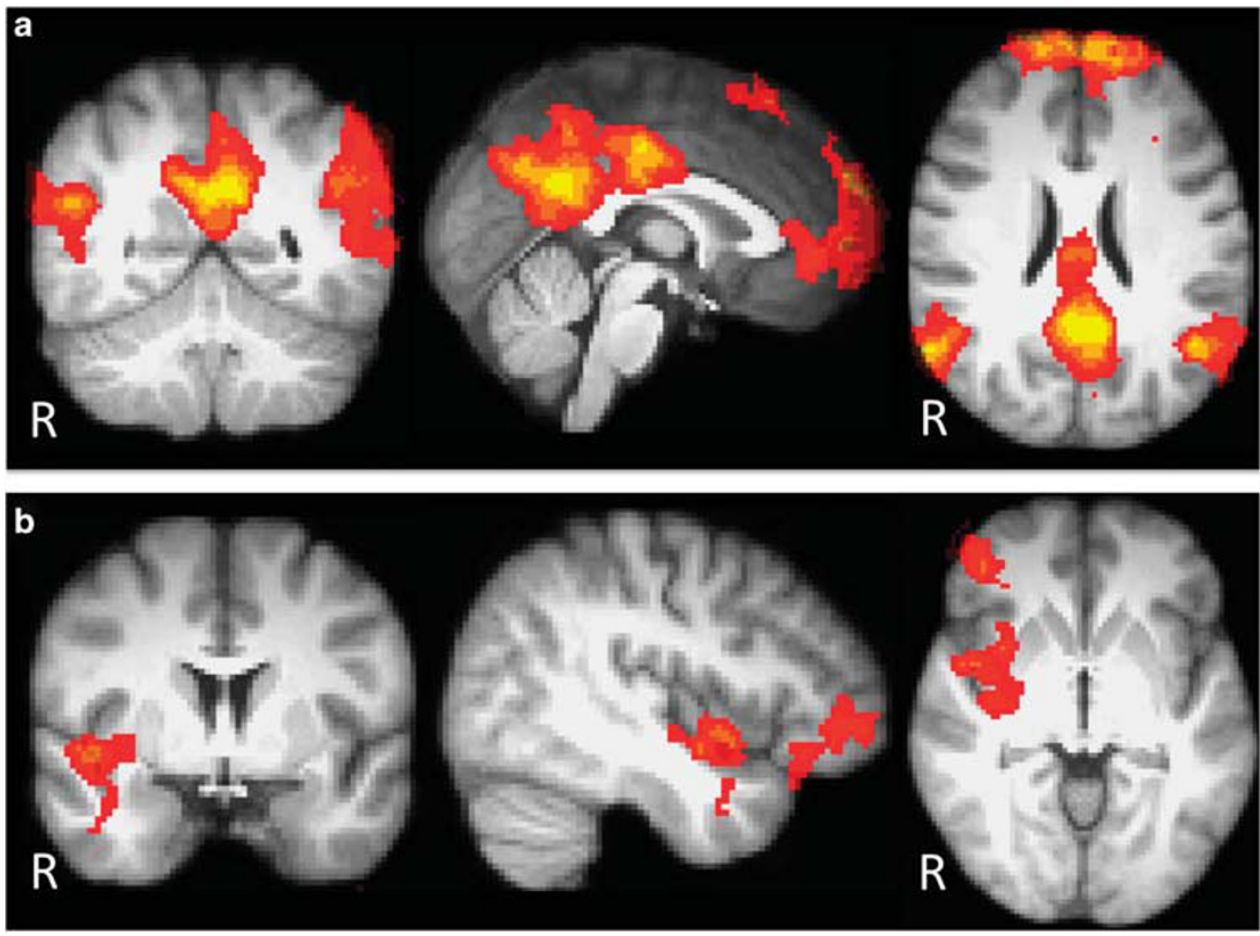

Figure I Brain reactivity to smoking cues. (a) On average, brain regions showing enhanced reactivity to smoking vs neutral cues include midline cortical structures as well as the bilateral angular gyri and insula. (b) Greater anterior insula-dorsal anterior cingulate cortex (Al-dACC) coupling at rest is associated with enhanced cue-reactivity in the visual cortex, right VLPFC, and putamen.

Table 2 Brain Reactivity to Smoking Cues Positively Correlated with Dorsal Anterior Insula-dACC Coupling

\begin{tabular}{|c|c|c|c|c|c|c|}
\hline Brain area & Brodmann area & $x$ & $y$ & $\mathbf{z}$ & Z-max & Voxels \\
\hline $\begin{array}{l}\text { Medial aspects of the lingual gyrus and fusiform gyrus; precuneus; and posterior } \\
\text { left superior parietal lobule }\end{array}$ & 17,18 & -14 & -58 & -4 & 3.53 & 1586 \\
\hline Right ventral lateral prefrontal cortex & 47,10 & 42 & 42 & -4 & 3.39 & 598 \\
\hline
\end{tabular}

Brain area and Brodmann area refer to the location of each cluster of contiguous voxels. MNI coordinates $(x, y, z)$ refer to the region of maximum cluster activation for each cluster. Z-max refers to the maximum $Z$-statistic in each cluster ( $\left.p_{\text {cluster corrected }}<0.05\right)$. Voxels refer to the total number of voxels within the cluster.

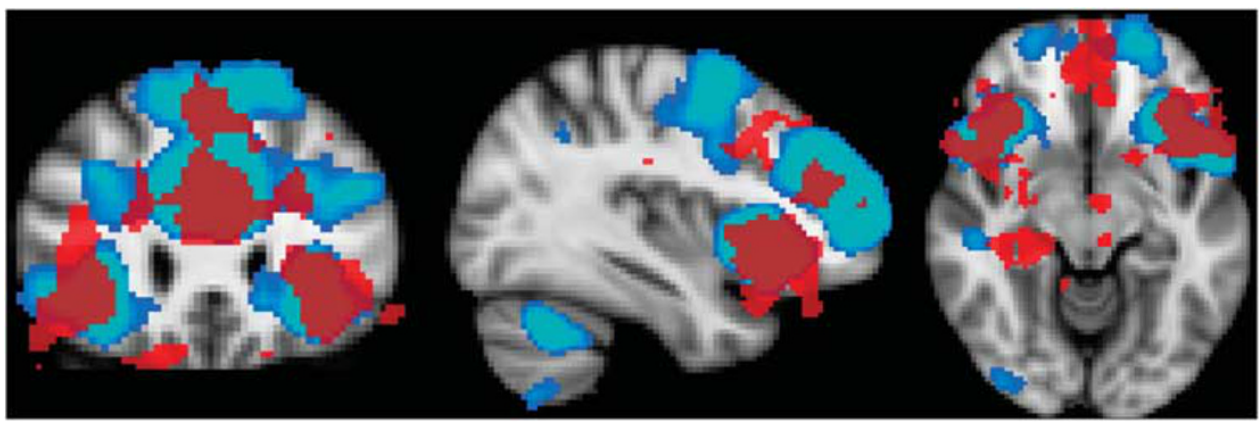

Figure 2 Cue-reactivity and the salience network. Blue overlay represents the salience network as defined at rest using independent component analysis. The red overlay represents the brain reactivity to smoking cues that corresponds with bilateral dorsal anterior insula reactivity to smoking cues.

On average, brain reactivity to smoking $v s$ neutral cues induced activity in the insula, bilateral angular gyri, and midline cortical brain regions (ACC, PCG, MPFC, and precuneus). This pattern of activation is the same that we reported in our previous work (Janes et al, 2013b), and was highlighted in a meta-analysis of smoking cue-reactivity 
(Engelman et al, 2012). These brain regions are consistently reactive to smoking cues across studies, and also overlap with the default mode network (DMN), which is a wellcharacterized functional brain network. In fact, we found significant correspondence between this cue-reactivity pattern and the DMN as defined at rest using ICA (fslcc, $r=0.44)$. The DMN is typically suppressed during cognitively demanding tasks and is associated with autobiographical memory and self-referential thought (Buckner and Carroll, 2007; Gusnard et al, 2001; Heatherton et al, 2006; Macrae et al, 2004; Moran et al, 2006). This finding not only suggests that smokers process smoking stimuli as personally relevant but also that cue-induced activation of the DMN engages 'self-referential thoughts' potentially related to ruminations related to drug use. When taking baseline AI-dACC connectivity strength into account, increased AIdACC coupling at rest was associated with greater cuereactivity in brain regions including the visual cortex, right VLPFC, and putamen. One possibility is that greater AIdACC coupling at rest represents facilitated communication within the SN, leading to enhanced processing of salient stimuli by brain regions associated with attention and action selection.

A recent meta-analysis revealed that there was substantial evidence that primary and secondary visual cortices typically react to drug-related stimuli (Hanlon et al, 2014), which may represent enhanced attention (Pratte et al, 2013) to the drug cues. Expanding this line of reasoning to the current findings suggests that smokers with enhanced AI-dACC coupling may attend more strongly to smoking cues. This idea of heightened attention is further supported by the observed right VLPFC activity, as this region is part of the ventral attention system, which is involved in the detection of behaviorally relevant sensory events (Corbetta et al, 2008; Corbetta and Shulman, 2002; Hampshire et al, 2010). One theory suggests that the right VLPFC acts as a site of inhibitory motor control (Aron et al, 2004; Verbruggen and Logan, 2009). However, others postulate that the right VLPFC may instead act as a 'circuit breaker' of ongoing cognitive processes when motivationally salient stimuli appear (Corbetta and Shulman, 2002). The right VLPFC is therefore thought to be part of a reorienting network that shifts attention toward behaviorally relevant stimuli, which supports the idea that stronger AI-dACC coupling may lead to greater attentional processing of smoking-related stimuli. Such right VLPFC activity also may be behaviorally relevant. For instance, obese individuals experienced right VLPFC activation in response to food images and this corresponded with total body fat, suggesting that right VLPFC activation to salient stimuli may be related to consumatory behaviors (Yokum et al, 2011). In contrast, the lack of behavioral responding to salient stimuli is associated with right VLPFC hypoactivity (De Ruiter et al, 2009). Collectively, these studies suggest that right VLPFC activity may represent the attentional shift toward salient stimuli necessary for behavioral responding. In the context of the current study, such right VLPFC activity to cues is enhanced specifically in those smokers with the greatest baseline AI-dACC coupling at rest suggests that inherent brain organization may facilitate this activity.

Dorsal AI-dACC coupling at rest was also associated with enhanced putamen reactivity to smoking cues, which is pertinent given the wealth of data implicating the putamen/ dorsal striatum in craving and drug abuse. In fact, increased dopamine release in the dorsal striatum is related to cocaine cue-induced craving in humans (Volkow et al, 2006) and drug seeking in rodents (Ito et al, 2002). We also reported that greater dorsal striatal volume is associated with enhanced smoking cue-induced craving (Janes et al, 2014b). Following chronic drug self-administration, the dorsal striatum has an increasingly prominent role in maintaining addiction (Porrino et al, 2004), and is thought to maintain habitual drug seeking (See et al, 2007) due to this region's role in facilitating behavioral responding to rewarding stimuli (Haruno and Kawato, 2005; Yamada et al, 2004). On a global basis, the dorsal striatum is associated with motivation, or 'the drive for action that leads one to work to obtain rewards' (Miller et al, 2014, page 1075), which, in relation to the current work may represent the drive to obtain smoking-related reward.

In a separate cohort of smokers, we showed that AIdACC connectivity is stable over $\sim 1$-h delay, and that AIdACC connectivity is not influenced by changes in craving or CO over this time period. While these analyses suggest that AI-dACC connectivity may be a trait, it is unclear whether AI-dACC connectivity is consistent over longer time delays and following changes in smoking status. However, it is tempting to speculate that greater AI-dACC connectivity may represent an underlying vulnerability for enhanced processing of salient stimuli, such as smoking cues, which gain salient properties due to their repeated pairing with nicotine (Caggiula et al, 2009). Such vulnerability may render those smokers with enhanced AI-dACC coupling even more likely to react to smoking cues, making abstinence difficult when in contact with these cues. Whether this is a pre-existing trait or is influenced by nicotine use remains to be determined. However, the fact that there was no relationship between AI-dACC connectivity and measures related to smoking history suggests that any influence of nicotine may be more nuanced than what is presented in the current study. Alternatively, research involving a larger cohort of smokers, thus capturing more variation than the present work, also may shed light on this issue. As the current study uses a limited sample, studies including more participants are also necessary to confirm our findings. Future work also is needed to determine what conditions can modulate AI-dACC connectivity and the subsequent effect on smoking cue-reactivity. Such research will allow the field to determine whether AI-dACC coupling is, in fact, a stable trait or whether treatments could be developed that modulate this circuit.

The current result suggests some regional specificity as AI-dACC interactions were related to cue-reactivity while mid and posterior insula interactions with the dACC were not. However, this does not preclude other insula circuits from playing a role in nicotine dependence. In fact, insula interactions with the ventral MPFC are associated with both difficulty identifying affective state and greater craving during nicotine withdrawal (Sutherland et al, 2013b), and nicotinic agents modulate the insula-amygala interactions in abstinent smokers (Sutherland et al, 2013). The idea that the insula plays a role in multiple facets of nicotine use is further supported by preclinical work as the insula is involved not only in cue-reactivity but also in nicotine 
intake and nicotine reinstatement following a priming dose (Forget et al, 2010; Pushparaj et al, 2013). Collectively, these studies implicate the insula in a range of processes associated with nicotine dependence and move the field toward a better understanding of how sub-regions, and various network interactions may support these functions.

An additional limitation of this research is that we focused only on the relationship between AI-dACC connectivity and smoking cues and we are unable to relate AI-dACC coupling with other forms of salience processing. It is unlikely that enhanced AI-dACC connectivity only facilitates reactivity to smoking cues, but may in fact impact salience processing more generally. While the pattern of brain activation suggests that the AI-dACC coupling may influence behavioral responding to smoking cues, we do not have a direct measure of smoking behavior to relate to these imaging findings. Nevertheless, the present findings confirm our a priori hypothesis that inherent connectivity between primary SN nodes impacts brain reactivity to smoking cues and suggests that enhanced baseline AIdACC functional connectivity may be a factor leading to greater engagement of attentional and motivational resources when processing salient stimuli such as smoking cues, which may ultimately impact relapse.

\section{FUNDING AND DISCLOSURE}

This work was supported by the National Institute on Drug Abuse Grant K01DA029645 (AJ). AP was supported by the Rossano Mind, Brain, and Behavior Pre-Doctoral Summer Fellowship at the McLean Hospital. The author declares no conflict of interest.

\section{REFERENCES}

Allman JM, Tetreault NA, Hakeem AY, Manaye KF, Semendeferi K, Erwin JM et al (2010). The von Economo neurons in frontoinsular and anterior cingulate cortex in great apes and humans. Brain Struct Funct 214: 5-6.

Andrews-Hanna JR, Snyder AZ, Vincent JL, Lustig C, Head D, Raichle ME et al (2007). Disruption of large-scale brain systems in advanced aging. Neuron 56: 924-935.

Aron AR, Robbins TW, Poldrack RA (2004). Inhibition and the right inferior frontal cortex. Trends Cogn Sci 8: 170-177.

Buckner RL, Carroll DC (2007). Self-projection and the brain. Trends Cogn Sci 11: 49-57.

Caggiula AR, Donny EC, Palmatier MI, Liu X, Chaudhri N, Sved AF (2009). The role of nicotine in smoking: a dual-reinforcement model. Nebr Symp Motiv 55: 91-109.

Corbetta M, Patel G, Shulman GL (2008). The reorienting system of the human brain: from environment to theory of mind. Neuron 58: $306-324$.

Corbetta M, Shulman GL (2002). Control of goal-directed and stimulus-driven attention in the brain. Nat Rev Neurosci 3: 215-229.

Cox LS, Tiffany ST, Christen AG (2001). Evaluation of the brief questionnaire of smoking urges (QSU-brief) in laboratory and clinical settings. Nicotine Tob Res 3: 7-16.

De Ruiter MB, Veltman DJ, Goudriaan AE, Oosteriaan J, Sjoerds Z, van den Brink W (2009). Response perseveration and ventral prefrontal sensitivity to reward and punishment in male problem gamblers and smokers. Neuropsychopharmacology 34: 1027-1038.
Dosenbach NU, Fair DA, Miezin FM, Cohen AL, Wenger KK, Dosenbach RA et al (2007). Distinct brain networks for adaptive and stable task control in humans. PNAS 104: 11073-11078.

Dosenbach NU, Visscher KM, Palmer ED, Miezin FM, Wenger KK, Kang HC et al (2006). A core system of the implementation of task sets. Neuron 50: 799-812.

Engelmann JM, Versace F, Robinson JD, Minnix JA, Lam CY, Cui Y et al (2012). Neural substrates of smoking cue reactivity: a metaanalysis of fMRI studies. Neuroimage 60: 252-262.

Fagerström KO (1978). Measuring degree of physical dependence to tobacco smoking with reference to individualization of treatment. Addict Behav 3: 235-241.

Ferguson SG, Shiffman S (2009). The relevance and treatment of cue- induced cravings in tobacco dependence. J Subst Abuse Treat 36: 235-243.

Forget B, Pushparaj A, Le Foll B (2010). Granular insular cortex inactivation as a novel therapeutic strategy for nicotine addiction. Biol Psychiatry 68: 265-271.

Gusnard DA, Akbudak E, Shulman GL, Raichle ME (2001). Medial prefrontal cortex and self-referential mental activity: relation to a default mode of brain function. PNAS 98: 4259-4262.

Hampshire A, Chamberlain SR, Monti MM, Duncan J, Owen AM (2010). The role of the right inferior frontal gyrus: inhibition and attentional control. Neuroimage 50: 1313-1319.

Hanlon CA, Dowdle LT, Naselaris T, Canterberry M, Cortes BM (2014). Visual cortex activation to drug cues: a meta-analysis of functional neuroimaging papers in addiction and substance abuse literature. Drug Alcohol Depend 143: 206-212.

Haruno M, Kawato M (2005). Different neural correlates of reward expectation and reward expectation error in the putamen and caudate nucleus during stimulus-action-reward association learning. J Neurophysiol 95: 948-959.

Heatherton TF, Wyland CL, Macrae CN, Demos KE, Denny BT, Kelley WM (2006). Medial prefrontal activity differentiates self from close others. Soc Cogn Affect Neurosci 1: 18-25.

Ito R, Dalley JW, Robbins TW, Everitt BJ (2002). Dopamine release in the dorsal striatum during cocaine-seeking behavior under the control of a drug-associated cue. J Neurosci 22: 6247-6253.

Janes AC, Farmer S, Frederick BB, Nickerson LD, Lukas SE (2014a). An increase in tobacco craving is associated with enhanced medial prefrontal cortex network coupling. PloS One 9: e88228.

Janes AC, Jensen JE, Farmer SL, Frederick BB, Pizzagalli DA, Lukas SE (2013a). GABA levels in the dorsal anterior cingulate cortex associated with difficulty ignoring smoking-related cues in tobacco-dependent volunteers. Neuropsychopharmacology 38: 1113-1120.

Janes AC, Nickerson LD, Frederick BB, Kaufman MJ (2012). Prefrontal and limbic resting state brain network functional connectivity differs between nicotine-dependent smokers and non-smoking controls. Drug Alcohol Depend 125: 252-259.

Janes AC, Park MT, Farmer S, Chakravarty MM (2014b). Striatal morphology is associated with tobacco cigarette craving. Neuropsychopharmacology 40: 406-411.

Janes AC, Pizzagalli DA, Richardt S, Frederick BB, Chuzi S, Pachas $\mathrm{G}$ et al (2010). Brain reactivity to smoking cues prior to smoking cessation predicts ability to maintain tobacco abstinence. Biol Psychiatry 67: 722-729.

Janes AC, Ross RR, Farmer S, Frederick BB, Nickerson LD, Lukas SE et al (2013b). Memory retrieval of smoking-related images induce greater insula activation as revealed by an fMRIbased delayed matching to sample task. Addict Biol; e-pub ahead of print 22 November 2013; doi: 10.1111/adb.12112.

Kelly RE, Alexopoulos GS, Wang Z, Gunning FM, Murphy CF, Morimoto SS et al (2010). Visual inspection of independent components: defining a procedure for artifact removal from fMRI data. J Neurosci Methods 189: 233-245. 
Macrae CN, Moran JM, Heatherton TF, Banfield JF, Kelly WM (2004). Medial prefrontal activity predicts memory for self. Cereb Cortex 14: 647-654.

Menon V (2011). Large-scale brain networks and psychopathology: a unifying triple network model. Trends Cogn Sci 15: 483-506.

Menon V, Uddin LQ (2010). Saliency, switching, attention and control: a network model of insula function. Brain Struct Funct 214: 655-667.

Miller EM, Shankar MU, Knutson B, McClure SM (2014). Dissociating motivation from reward in human striatal activity. J Cogn Neurosci 26: 1075-1084.

Moran JM, Heatherton TF, Kelley WM (2006). Modulation of cortical midline structures by implicit and explicit self- relevance evaluation. Soc Neurosci 4: 197-211.

Naqvi NH, Bechara A (2009). The hidden island of addiction: the insula. Trends Neurosci 32: 56-67.

Naqvi NH, Gaznick N, Tranel D, Bechara A (2014). The insula: a critical neural substrate for craving and drug seeking under conflict and risk. Ann NY Acad Sci 1316: 53-70.

Naqvi NH, Rudrauf D, Damasio H, Bechara A (2007). Damage to the insula disrupts addiction to cigarette smoking. Science 315: 531-534.

Nelson SM, Dosenbach NU, Cohen AL, Wheeler ME, Schlagger BL, Petersen SE (2010). Role of the anterior insula in task-level control and focal attention. Brain Struct Funct 214: 669-680.

Oppenheim AV, Schafer RW (1975). Digital Signal Processing. Prentice-Hall International: London1975, pp 242.

Ploran EJ, Nelson SM, Velanova K, Donaldson DI, Petersen SE, Wheeler ME (2007). Evidence accumulation and the moment of recognition: dissociating perceptual recognition processes using fMRI. J Neurosci 27: 11912-11924.

Porrino LJ, Lyons D, Smith HR, Daunais JB, Nader MA (2004). Cocaine self-administration produces a progressive involvement of limbic, association, and sensorimotor striatal domains. J Neurosci 24: 3554-3562.

Pratte MS, Ling S, Swisher JD, Tong F (2013). How attention extracts objects from noise. J Neurophysiol 110: 1346-1356.
Pushparaj A, Hamani C, Yu W, Shin DS, Kang B, Nobrega JN et al (2013). Electrical stimulation of the insula regions attenuates nicotine-taking and nicotine-seeking behaviors. Neuropsychopharmacology 38: 690-698.

See RE, Elliott JC, Feltenstein MW (2007). The role of dorsal vs ventral striatal pathways in cocaine-seeking behavior after prolonged abstinence in rats. Psychopharmacology 194: 321-331.

Seeley WW, Menon V, Schatzberg AF, Keller J, Glover GH, Kenna $\mathrm{H}$ et al (2007). Dissociable intrinsic connectivity networks for salience processing and executive control. J Neurosci 27: 2349-2356.

Sutherland MT, Carroll AJ, Salmeron BJ, Ross TJ, Hong LE, Stein EA (2013). Down-regulation of amygdala and insula functional circuits by varenicline and nicotine in abstinent cigarette smokers. Bio Psychiatry 74: 538-546.

Sutherland MT, Carroll AJ, Salmeron BJ, Ross TJ, Stein EA (2013b). Insula's functional connectivity with ventromedial prefrontal cortex mediates the impact of trait alexithymia on state tobacco craving. Psychopharmacology 228: 143-155.

Verbruggen F, Logan GD (2009). Automaticity of cognitive control: goal priming in response-inhibition paradigms. J Exp Psychol Learn Mem Cogn 35: 1381-1388.

Volkow ND, Wang G, Telang F, Fowler JS, Logan J, Childress AR et al (2006). Cocaine cues and dopamine in dorsal striatum: mechanism of craving in cocaine addiction. J Neurosci 26: 65836588.

Wager TD, Barret LF (2004): From affect to control: functional specialization of the insula, motivation and regulation. Available at PsychExtra http://www.columbia.edu/cu/psychology/tor/. accessed on 20 November 2012.

Yamada H, Matsumoto N, Kimura M (2004). Tonically active neurons in the primate caudate nucleus and putamen differentially encode instructed motivational outcomes of action. J Neurosci 24: 3500-3520.

Yokum S, Ng J, Stice E (2011). Attentional bias to food images associated with elevated weight and future weight gain: an fMRI study. Obesity 19: 1775-1783.

Supplementary Information accompanies the paper on the Neuropsychopharmacology website (http://www.nature.com/npp) 